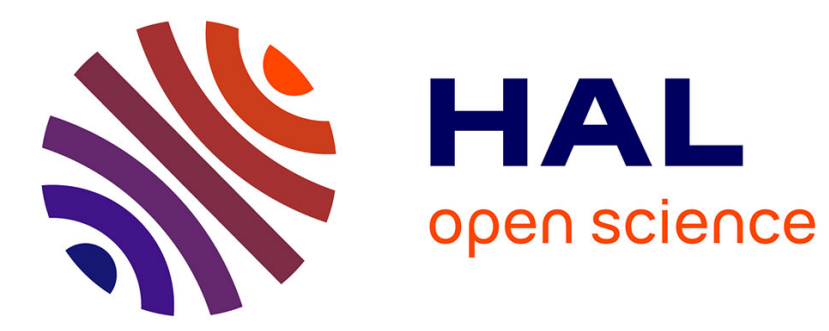

\title{
Piezomagnetic tensors symmetries: an unifying tentative approach
}

\author{
Thibaut Weller, Giuseppe Geymonat
}

\section{To cite this version:}

Thibaut Weller, Giuseppe Geymonat. Piezomagnetic tensors symmetries: an unifying tentative approach. Configurational Mechanics, A. A. Balkema Publishers, 13p., 2004. hal-00797835

\section{HAL Id: hal-00797835 \\ https://hal.science/hal-00797835}

Submitted on 7 Mar 2013

HAL is a multi-disciplinary open access archive for the deposit and dissemination of scientific research documents, whether they are published or not. The documents may come from teaching and research institutions in France or abroad, or from public or private research centers.
L'archive ouverte pluridisciplinaire HAL, est destinée au dépôt et à la diffusion de documents scientifiques de niveau recherche, publiés ou non, émanant des établissements d'enseignement et de recherche français ou étrangers, des laboratoires publics ou privés. 


\title{
Piezomagnetic tensors symmetries : an unifying tentative approach
}

\author{
T. Weller, G. Geymonat \\ Laboratoire de Mécanique et Génie Civil, UMR-CNRS 5508, \\ c.c. 048, Université Montpellier II, Place Eugène Bataillon \\ 34095 Montpellier Cedex 5, France \\ thibaut.weller@univ-montp2.fr
}

\begin{abstract}
Traditionally, one inquires as to what is the form of a physical property tensor invariant under the point group of a crystal. For example, one finds listings showing 16 distinct forms of the piezomagnetic tensor that can arise in a crystal. Alongside each of these 16 distinct forms is listed the point groups which give rise to that specific form (see Birss (1966) for example). This is a classification of the form of piezomagnetic tensors in crystals. We claim however that this classification is quite ambiguous in the sense that it hides the very difference between microscopic and macroscopic symmetries. The topic of the paper is the both a clarification of the difference between material and behaviour symmetries and a classification of piezomagnetic tensors in behaviour equivalences classes, found to be 15 .
\end{abstract}

\section{Introduction}

All physical properties of crystals are related to their structure. This elementary observation is at the start of crystal physics. However, as one moves away from microscopic field to macroscopic behavior, the tools, techniques and models evolve sufficiently so that, finally, they are not any more considered in their continuity. For example, with regard to elasticity, for which symmetries play an eminent role in the mathematical formulation of its principles, the crystallographic point of view of matter's properties is often forsaken and, most of the time, the macroscopic prospect only remains. Within this framework, one finds in Huo \& Del Piero (1991) the first attempt to classify hyperelastic tensors according to their type of symmetry by building equivalence classes. This classification was then definitively established in Forte \& Vianello (1996) and, with regard to photoelastic tensors, in Forte \& Vianello (1997). See Forte \& Vianello (1996) for a detailed history of the step in elasticity.

The method initiated in these two references is particularly adapted to all types of mechanical behavior. In Geymonat \& Weller (2002) it is extended to piezoelectricity. The step is here considered in the micro-macro continuity and applied to piezomagnetism.

Piezomagnetic crystals are characterized by the appearance of a magnetic moment under the application of a stress. In addition to the usual symmetry operations which one meets in crystallography (namely: orthogonal transformations, i.e. elements of the orthogonal group denoted $\mathbf{O}(\mathbf{3})$ ) we consider a generally denoted $\mathfrak{R}$ operation whose action is to change the sign of the moment. This is why it is often associated to time-reversal (cf. Landau \& Lifchitz, 1969; Laughlin et al., 2000; Sivardière, 1995). The symmetry operations which act on piezomagnetic crystals thus require the definition of the extended orthogonal group denoted $\mathbf{O}^{\mu}(\mathbf{3})$ (cf. Sirotine \& Chaskolskaïa (1984) as well as (9) formula).

The common background between microscopic and macroscopic approaches is the construction of equivalence classes. On the one hand, each crystalline structure is associated to a subgroup of the (eventually extended) orthogonal group : its point group. Of course, one can also build its Bravais lattice or its space group, which correspond to other kinds of equivalence classes. On the other hand, if we consider that tensors accurately translate the physical properties of matter, we are brought to build actions which, as for crystals, will enable us to associate a subgroup of $\mathbf{O ( 3 )}$, or eventually $\mathbf{O}^{\mu}(\mathbf{3})$, to a given tensor. This subgroup is the tensor symmetry class. We will use the Cauchy-Born hypothesis to bind these two types of symmetry.

In $\S 2$ we recall traditional results of crystallography by using the mathematical tools developed in Pitteri \& Zanzotto (1996, 1998, 2003). Crystal lattices are then rigorously classified in equivalence classes. Those can be arithmetic or geometric, then defining respectively the 14 Bravais lattices and the 7 crystal systems. Crystals are then ranged in 32 point groups and 230 space groups. These numbers increase to 90 point groups and 1421 space groups in the piezomagnetic crystals case, due to the additional time-reversal operation.

These results are very traditional. We nevertheless insist on the manner that is used to define and range these equivalence classes, because these methods are then transposed to piezomagnetic tensors by the use of the Cauchy-Born hypothesis, introduced in $\S 3$. Piezomagnetic tensors are axial tensors. We show in $\S 3.3$ the way we consider the action of the extended orthogonal group on such tensors. It is shown in particular that, via an isomorphism, piezomagnetic tensors can be viewed as piezoelectric ones. The mathematical tools which make it possible to exhaust symmetry classes of such tensors are the harmonic and Cartan decompositions.

Harmonic decomposition maps a tensor of any order into a $n$-uplet of totally symmetric tensors (invariant by any permutations of its indexes) which are also traceless (the trace with respect to any pair of indexes is zero). These traceless totally symmetric tensors are then identified via an isomorphism to harmonic homogeneous polynomials (within the Laplacian sense) of corresponding degree. This is why a traceless totally symmetric tensor is known as harmonic (cf. Forte \& Vianello, 1996, 1997; Schouten, 1954; Spencer, 1970). As to Cartan decomposition (cf. Golubitsky et al., 1998), it is an operation by which harmonic polynomials are rewritten in spaces where symmetry operations become elementary. 
T. Weller \& G. Geymonat

Each piezomagnetic tensor is then associated to a subgroup of $\mathbf{O}^{\mu}(3)$, i.e. to a kind of behavior which is connected, in $\S 4$, to the magnetic point groups which give it birth. It is in particular shown that these point groups should not be classified in 16 but in 15 symmetry classes.

\section{Preliminaries: formulation of microscopic equivalence classes}

\subsection{Crystal lattices}

Mathematical modelings of crystal lattices and, in general, of crystallography, have been explored by a rich and various literature (see for instance Coxeter, 1989; Pitteri \& Zanzotto, 1996, 1998, 2003; Weigel, 1972; Yale, 1968). We here take the way as it has been opened by Pitteri \& Zanzotto (1996, 1998, 2003) within the framework of phase transition in solids mechanics.

We note $\mathbf{L i n}$ the space of second-order tensors, $\mathbf{I}$ the unit element in $\mathbf{L i n}$ and $\mathbf{L}^{t}$ the transpose of the element $\mathbf{L} \in \mathbf{L i n}$. We recall that $\mathbf{O ( 3 )}=\left\{\mathbf{L} \in \mathbf{L i n}: \mathbf{L}^{t}=\mathbf{L}^{t} \mathbf{L}=\mathbf{I}\right\}$ is the space of orthogonal transformations and that the subgroup of all elements of $\mathbf{O}(\mathbf{3})$ with determinant equal to 1 (or rotations group) is denoted $\mathbf{S O}(\mathbf{3})$.

Let $\mathbb{R}^{3}$ be the three-dimensional Euclidean vector space. Three linearly independent vectors $\mathbf{e}_{a}(a=1,2,3)$ of $\mathbb{R}^{3}$ define a basis denoted $\left\{\mathbf{e}_{a}\right\}=\left(\mathbf{e}_{1}, \mathbf{e}_{2}, \mathbf{e}_{3}\right)$. The convention of summation over repeated indexes being understood, we define the (simple) lattice $\mathcal{R}\left(\left\{\mathbf{e}_{a}\right\}\right)$ by:

$$
\mathcal{R}\left(\left\{\mathbf{e}_{a}\right\}\right):=\left\{\mathbf{x} \in \mathbb{R}^{3}: \mathbf{x}=M^{a} \mathbf{e}_{a}\right.
$$

$$
\left.a=1,2,3, M^{a} \in \mathbb{Z}\right\} .
$$

We also say that the 3 linearly independent vectors $\mathbf{e}_{a}(a=1,2,3)$ are the basis vectors of $\mathcal{R}\left(\left\{\mathbf{e}_{a}\right\}\right)$. The parallelepiped $\mathcal{P}\left(\left\{\mathbf{e}_{a}\right\}\right)$ spanned by $\mathbf{e}_{a}(a=1,2,3)$ is called the unit cell of $\mathcal{R}\left(\left\{\mathbf{e}_{a}\right\}\right)$.

It is well known that lattices can be ranged in 7 crystal systems or 14 Bravais lattices. These two types of classification correspond to two types of equivalence classes: "geometrical" equivalence classes and "arithmetic" equivalence classes. These two kinds of equivalence classes arise on the one hand from geometrical actions (namely: elements $\mathbf{Q}$ of $\mathbf{O}(\mathbf{3})$ ) and on the other hand from arithmetic actions (elements $m$ of $G L(3, \mathbb{Z})$, the space of $3 \times 3$ invertible unimodular matrices with integral entries). We thus define in the following the way these elements act on lattices and then build the equivalence classes which result from these actions.

An element $\mathbf{Q} \in \mathbf{O}(\mathbf{3})$ acts on $\left\{\mathbf{e}_{a}\right\}$ in the following way:

$$
\mathbf{Q}\left\{\mathbf{e}_{a}\right\}:=\left(\mathbf{Q} \mathbf{e}_{1}, \mathbf{Q} \mathbf{e}_{2}, \mathbf{Q} \mathbf{e}_{3}\right)=\left\{\mathbf{Q} \mathbf{e}_{a}\right\}
$$

As to arithmetic actions $m=\left(m_{a}^{b}\right) \in G L(3, \mathbb{Z})$, they are defined by:

$$
m\left\{\mathbf{e}_{a}\right\}:=\left(m_{1}^{b} \mathbf{e}_{b}, m_{2}^{b} \mathbf{e}_{b}, m_{3}^{b} \mathbf{e}_{b}\right)=\left\{m_{a}^{b} \mathbf{e}_{b}\right\} .
$$

These actions are quite different: notice that elements $\mathbf{Q} \in \mathbf{O}(\mathbf{3})$ act on each basis vector independently, while it is not the case of elements $m \in G L(3, \mathbb{Z})$.

Of course, a lattice is not attached to a unique basis. A lattice basis is only determined up to arithmetic transformations. More precisely, let $\left\{\mathbf{e}_{a}\right\}$ and $\left\{\mathbf{f}_{a}\right\}$ be two basis of $\mathbb{R}^{3}$, we have:

$$
\mathcal{R}\left(\left\{\mathbf{e}_{a}\right\}\right)=\mathcal{R}\left(\left\{\mathbf{f}_{a}\right\}\right)
$$

$$
\Longleftrightarrow \exists m \in G L(3, \mathbb{Z}),\left\{\mathbf{f}_{a}\right\}=m\left\{\mathbf{e}_{a}\right\}
$$

This invariance property is not the one which enables us to build interesting equivalence classes, but it is at the source of the various types of lattice symmetries.

We thus call geometrical holoedry $P\left(\left\{\mathbf{e}_{a}\right\}\right)$ of $\mathcal{R}\left(\left\{\mathbf{e}_{a}\right\}\right)$ the closed and finite subgroup of all orthogonal transformations $\mathbf{Q} \in \mathbf{O ( 3 )}$ leaving $\mathcal{R}\left(\left\{\mathbf{e}_{a}\right\}\right)$ unchanged. It is written:

$$
P\left(\left\{\mathbf{e}_{a}\right\}\right):=\left\{\mathbf{Q} \in \mathbf{O}(\mathbf{3}): \mathbf{Q}\left\{\mathbf{e}_{a}\right\}=m\left\{\mathbf{e}_{a}\right\}\right.
$$$$
m \in G L(3, \mathbb{Z})\}
$$

As to the lattice group (or arithmetic holohedry), it is defined by: 


$$
L\left(\left\{\mathbf{e}_{a}\right\}\right):=\left\{m \in G L(3, \mathbb{Z}): m\left\{\mathbf{e}_{a}\right\}=\mathbf{Q}\left\{\mathbf{e}_{a}\right\},\right.
$$

Roughly speaking, the geometric holohedry represents the "macroscopic family", while the lattice group informs about the manner of laying out triply periodically points in space.

Taking into account that

$$
P\left(m\left\{\mathbf{e}_{a}\right\}\right)=P\left(\left\{\mathbf{e}_{a}\right\}\right) \text { if } m \in G L(3, \mathbb{Z}),
$$

$$
P\left(\mathbf{Q}\left\{\mathbf{e}_{a}\right\}\right)=\mathbf{Q} P\left(\left\{\mathbf{e}_{a}\right\}\right) \mathbf{Q}^{t} \text { if } \mathbf{Q} \in \mathbf{O}(\mathbf{3}),
$$

we see that $P\left(\left\{\mathbf{e}_{a}\right\}\right)$ only depends on the lattice $\mathcal{R}\left(\left\{\mathbf{e}_{a}\right\}\right)$ itself, and not on its basis. Since $P\left(\left\{\mathbf{e}_{a}\right\}\right)$ describes geometric properties of $\mathcal{R}\left(\left\{\mathbf{e}_{a}\right\}\right)$ regarded as identical in any (geometrically) equivalent lattice, it is natural to put the initial $\mathbb{R}^{3}$ basis aside. Thus, rather than the holohedry $P\left(\left\{\mathbf{e}_{a}\right\}\right)$, we consider its equivalence class in $\mathbf{O ( 3 )}$. These equivalence classes are the crystal systems. Therefore, we say that two lattices are (geometrically) equivalent when their holohedries are conjugate in $\mathbf{O}(\mathbf{3})$, which means that they belong to the same crystal system. There are 7 such equivalence classes, widely known under the following denomination: triclinic, monoclinic, orthorhombic, tetragonal, trigonal, hexagonal and cubic.

We also have:

$$
L\left(\mathbf{Q}\left\{\mathbf{e}_{a}\right\}\right)=L\left(\left\{\mathbf{e}_{a}\right\}\right) \text { if } \mathbf{Q} \in \mathbf{O}(\mathbf{3})
$$

$$
L\left(m\left\{\mathbf{e}_{a}\right\}\right)=m^{-1} L\left(\left\{\mathbf{e}_{a}\right\}\right) m \text { if } m \in G L(3, \mathbb{Z}) .
$$

That means that an orthogonal transformation does not modify the lattice group $L\left(\left\{\mathbf{e}_{a}\right\}\right)$. Moreover, a change of lattice basis transforms $L\left(\left\{\mathbf{e}_{a}\right\}\right)$ into a conjugate within $G L(3, \mathbb{Z})$. Thus, it will be said that two lattices $\mathcal{R}\left(\left\{\mathbf{e}_{a}\right\}\right)$ and $\mathcal{R}\left(\left\{\mathbf{f}_{a}\right\}\right)$ (or two lattice basis $\left\{\mathbf{e}_{a}\right\}$ and $\left.\left\{\mathbf{f}_{a}\right\}\right)$ are arithmetically equivalent if their lattice groups are conjugate within $G L(3, \mathbb{Z})$. They then belong to the same Bravais class and we write $\left\{\mathbf{e}_{a}\right\} \stackrel{\mathbb{Z}}{\sim}\left\{\mathbf{f}_{a}\right\}$.

The arithmetic criterion is more precise than the data of an equivalence class in $\mathbf{O ( 3 )}$. The reason is that conjugacy within $G L(3, \mathbb{Z})$ is more stringent than within $\mathbf{O}(\mathbf{3})$. Thus, a geometric holohedry $P \subset \mathbf{O}(\mathbf{3})$ determines most of the time various lattice groups (the 14 Bravais lattices are obtained from the 7 crystal systems by introducing a node point at the center of the unit cell or at the center of the cell faces).

\subsection{Magnetic crystals as bicolor multilattices}

We here consider the three-dimensional real affine space $\mathbb{A}^{3}$. The subgroup of affine isometries of $\mathbb{A}^{3}$ is denoted $E(3)$. Once an origin 0 is chosen, any isometry $e \in E(3)$ can be represented by a couple $(\mathbf{t}, \mathbf{Q})$ where $\mathbf{t}$ is the vector from 0 to $e(0)$ and where $\mathbf{Q} \in \mathbf{O}(\mathbf{3})$.

Now, studying magnetic crystals, we need to introduce an additional operation that will be seen as reversing the sign of the magnetic moment when opering. This operation is generally denoted $\mathfrak{R}$ and can be regarded as reversing the course of time (not its sign) (cf. Landau \& Lifchitz, 1969; Laughlin et al., 2000; Sivardière, 1995; Sirotine \& Chaskolskaïa, 1984). This internal degree of freedom is the reason why one often speaks of black and white symmetry (see Shubnikov, 1964). Each color then represents the sign of the magnetic moment.

We therefore consider in the following the extended orthogonal group (cf. Sirotine \& Chaskolskaïa, 1984) of all orthogonal and antiorthogonal (i.e. associated to time-reversal) operations:

$$
\mathbf{O}^{\mu}(\mathbf{3}):=\mathbf{O}(\mathbf{3}) \times\{ \pm 1\} .
$$

An $\mathbf{O}^{\mu}(\mathbf{3})$ element is thus a couple $(\mathbf{Q}, \delta)$ where $\mathbf{Q}$ is an orthogonal transformation associated (if $\left.\delta=-1\right)$ or not (if $\left.\delta=1\right)$ to time-reversal. Let $(\mathbf{Q}, \delta)$ and $(\mathbf{R}, \theta)$ be two elements of $\mathbf{O}^{\mu}(\mathbf{3})$. The composition law in $\mathbf{O}^{\mu}(\mathbf{3})$ is given by

$$
(\mathbf{Q}, \delta)(\mathbf{R}, \theta)=(\mathbf{Q R}, \delta \theta) .
$$

Moreover, for later use (cf. relation (47) for instance), we denote ${ }^{\mu} \mathbb{I d}$ the subgroup $\{(\mathbf{I},+1),(-\mathbf{I},+1)\}$. We also denote $E^{\mu}(3)$ the set of all affine isometries and anti-isometries of $\mathbb{A}^{3}$, i.e. the set of all couples $(\mathbf{t}, \mathbf{Q}) \in \mathbb{R}^{3} \times \mathbf{O}^{\mu}(\mathbf{3})$.

Following Pitteri \& Zanzotto (2003), it is a simple matter of notation to extend to the magnetic case a translate of a simple lattice:

$$
\mathcal{L}\left(P^{ \pm}, \mathbf{e}_{a}\right):=P^{ \pm}+\mathcal{R}\left(\left\{\mathbf{e}_{a}\right\}\right) \subset \mathbb{A}^{3},
$$


where $P^{ \pm}$stands for the sign of the spin attached to the particle $P$ in $\mathbb{A}^{3}$. It is then clear that $\Re P^{+}=P^{-}$and vice-versa. This simply means: when reversing the course of time, the sign of the spin attached to the particle $P \in \mathbb{A}^{3}$ changes.

Then, according to Proposition 11.1 of Pitteri \& Zanzotto (2003), we can also define a magnetic crystal $\mathcal{M}^{\mu}$ as the subset of $\mathbb{A}^{3}$ such that

$$
\begin{aligned}
\mathcal{M}^{\mu} & =\mathcal{M}^{\mu}\left(P_{0}^{ \pm}, \cdots, P_{n-1}^{ \pm}, \mathbf{e}_{a}\right) \\
& :=\cup_{i=0}^{n-1} \mathcal{L}\left(P_{i}^{ \pm}, \mathbf{e}_{a}\right) .
\end{aligned}
$$

We adress the reader to Pitteri \& Zanzotto (2003) for further details.

\subsection{Space and point groups}

To get informations about the crystal structure, we introduce two types of symmetries, as in $\S 2.2$. Classically, they are known as point group symmetries and space group symmetries (this latter takes into account the translations).

We call magnetic space group of a multilattice $\mathcal{M}^{\mu}\left(P_{i}^{ \pm}, \mathbf{e}_{a}\right)$ the group denoted by $\mathcal{S}\left(\mathcal{M}^{\mu}\right)$ and defined as follows:

$$
\mathcal{S}\left(\mathcal{M}^{\mu}\right):=\left\{e \in E^{\mu}(3): e\left(\mathcal{M}^{\mu}\right)=\mathcal{M}^{\mu}\right\} .
$$

We then say that $e \in \mathcal{S}\left(\mathcal{M}^{\mu}\right)$ is an affine symmetry of the multilattice $\mathcal{M}^{\mu}$. As in $(7)_{2}$ and $(8)_{2}$ we have

$$
\mathcal{S}\left(e \mathcal{M}^{\mu}\right)=e \mathcal{S}\left(\mathcal{M}^{\mu}\right) e^{-1}
$$

Therefore, an affine symmetry of $\mathcal{M}^{\mu}$ changes $\mathcal{S}\left(\mathcal{M}^{\mu}\right)$ into a conjugate in $E^{\mu}(3)$.

We now consider the group $\mathcal{P}\left(\mathcal{M}^{\mu}\right)$ collecting all (anti)orthogonal transformations $\mathbf{Q} \in \mathbf{O}^{\mu}(\mathbf{3})$ preserving $\mathcal{M}^{\mu}$ when coupled with a suitable translation. We call $\mathcal{P}\left(\mathcal{M}^{\mu}\right)$ the magnetic point group of $\mathcal{M}^{\mu}$.

The following result is known for a long time, we extract it from Bhagavantam (1966) (see also Birss (1966 and Sivardière (1995)) and formulate it as Theorem 11.5 in Pitteri\&Zanzotto (2003) for the non magnetic case:

Theorem 1. There are 1421 conjugacy classes of magnetic space groups in the set of all the orientation-preserving (anti)affine transformations of $\mathbb{A}^{3}$.

There are 90 conjugacy classes of magnetic point groups in $\boldsymbol{O}^{\mu}(\mathbf{3})$.

\section{A macroscopic point of view: tensors symmetry classes}

\subsection{A bridge: the Cauchy-Born hypothesis}

The Cauchy-Born hypothesis is, roughly speaking, the statement that lattices vectors behave as material vectors. It simply means that when a crystal in the reference configuration with lattice $\mathcal{R}\left(\left\{\mathbf{e}_{a}^{0}\right\}\right)$ experiences a homogeneous deformation whose gradient is $\mathbf{F}$, we have:

$$
\mathbf{e}_{a}=\mathbf{F} \mathbf{e}_{a}^{0}
$$

where $\mathbf{e}_{a}$ are the lattice vectors of the crystal in the deformed configuration.

This quite simple hypothesis (whose validity is discussed in Pitteri \& Zanzotto (2003)) allows us to transfer symmetry properties studied up to now to the macroscopic level, then passing from geometrical to constitutive symmetry.

To this aim we first take for granted that the structure of crystals can be described by means of a simple lattice in all their allowed configurations. Second, we assume that the free energy $w$ per unit cell of a deformable crystalline body whose current configuration is a simple lattice $\mathcal{R}\left(\left\{\mathbf{e}_{a}\right\}\right)$ can be written as (in the following, we neglect the temperature $\theta$ ):

$w=\bar{w}\left(\mathbf{e}_{a}\right)$.

We just have to write that

$$
w=\bar{w}\left(\mathbf{e}_{a}\right)=\bar{w}\left(\mathbf{F e}_{a}^{0}\right)=\widetilde{w}(\mathbf{F})=\hat{w}(\mathbf{C})^{1},
$$


to obtain, by classical arguments (see Pitteri \& Zanzotto (2003) or Bhattacharya (2002) for example)

$$
\hat{w}(\mathbf{C})=\hat{w}\left(\mathbf{H}^{t} \mathbf{C H}\right)
$$

for all symmetric and positive definite tensor $\mathbf{C}$ and for all $\mathbf{H}$ in $G L(3, \mathbb{Z})$.

\subsection{Application to piezomagnetism}

For the sake of simplicity, we won't speak about the Pitteri-Ericksen neighborhoods in the following and we will just focus on the main step of the derivation of macroscopic equivalence classes (see Pitteri \& Zanzotto (2003) for further details).

In the piezomagnetic case, the energy is a function of the strain tensor $\mathbf{E}$ (whose Cauchy-Green tensor is $\mathbf{C}$ ) and of the magnetization polar $^{2}$ vector $\mathbf{b}$ :

$$
w=\tilde{w}(\mathbf{E}, \mathbf{b})=\hat{w}(\mathbf{C}, \mathbf{b}) .
$$

Restricting the invariance relation in (18) to the orthogonal transformations, we have:

$$
\hat{w}(\mathbf{C}, \mathbf{b})=\hat{w}\left(\mathbf{Q}^{t} \mathbf{C Q}, \operatorname{det}(\mathbf{Q}) \mathbf{Q b}\right),
$$

$$
\forall \mathbf{Q} \in P\left(\left\{\mathbf{e}_{a}^{0}\right\}\right),
$$

because $\mathbf{b}$ is an axial vector (see relation (27)). Moreover, as the sign of the magnetization vector $\mathbf{b}$ directly depends on the course of time, we have $\mathfrak{R} \mathbf{b}=-\mathbf{b}$ and therefore:

$$
\tilde{w}(\mathbf{C}, \mathbf{b})=\tilde{w}(\mathbf{C},-\mathbf{I} \Re \mathbf{b}) .
$$

Now, the piezomagnetic tensor $\mathbf{p}$ is given by:

$$
\mathbf{p}=\frac{\partial^{2} \hat{w}}{\partial \mathbf{C} \partial \mathbf{b}}
$$

So,in the linear case, since $\mathbf{E}=\frac{1}{2}(\mathbf{C}-\mathbf{I})$, relations (20) and (21) lead to:

$$
\begin{aligned}
\mathbf{Q} \mathbf{p}[\mathbf{b}] \mathbf{Q}^{t} & =\operatorname{det}(\mathbf{Q}) \mathbf{p}[\mathbf{Q} \mathbf{b}], \\
\mathbf{Q}^{t} \mathbf{p}[\mathbf{E}] & =\mathbf{p}\left[\mathbf{Q}^{t} \mathbf{E} \mathbf{Q}\right],
\end{aligned}
$$

for all $\mathbf{Q}$ in $P\left(\left\{\mathbf{e}_{a}^{0}\right\}\right)$, all $\mathbf{b}$ in $\mathbb{R}^{3}$ and all second order symmetric tensor $\mathbf{E}$. Furthermore,

$$
\Re \mathbf{p}=\mathbf{- I} \mathbf{p}=-\mathbf{p}
$$

which means that the piezomagnetic tensor is odd according to time-reversal (this is meaningless in $(23)_{2}$ because in this relation, the piezomagnetic tensor acts on $\mathbf{E}$ which do not depend on the course of time).

It is important here to compare $(23)_{1}$ and (24) to the action that will arise in (32). It is clearly show how, in this way, some of the symmetry elements present in a crystal can be brought across the microscopic level to the macroscopic one, where another equivalence classes can be built. In fact, $(23)_{1}$ says that each element of the geometrical holoedry of a studied crystal is an element of the symmetry group of the piezomagnetic tensor of this crystal. This has to be compared to the Neumann's Principle, which states that "the symmetry group of any property of a crystal must include the point group of this last". We emphasize on the fact that all hypothesis that have been made since we introduced the Cauchy-Born hypothesis concern the crystal viewed as a lattice. The point groups therefore cannot appear in this approach.The important fact, anyway, is how the $\star$ action that will be introduced in the next section, and which is of fundamental use in the tensors symmetries study, naturally arises from the crystal structure via the Cauchy-Born hypothesis (see also Pitteri \& Zanzotto $\S 6.6 .2)$.

\footnotetext{
${ }^{2}$ See $\S 3.3$ for further details on polar variables.
} 


\subsection{Polar and axial variables}

The distinction between polar and axial variables is related to the need of defining, when necessary, an orientation in the three dimensional Euclidean space. We shall speak of polar variable when it is defined without ambiguity by the nature. In particular, its sign does not depend on any anthropomorphic convention, nor on any reference to a basis. On the other hand, a variable is known as axial if its sign cannot be fixed without a human reference to space orientation. An axial variable is only defined in an oriented affine space.

In order to formalize this, we consider a tensor of any order noted $\mathbf{T}$. Its components in a given basis $R=(O ; \mathbf{u}, \mathbf{v}, \mathbf{w})$ are denoted $\mathbf{T} \ldots i j k \ldots$ Let $\mathbf{Q} \in \mathbf{O}(\mathbf{3})$ be an orthogonal transformation. In the new basis $R^{\prime}=(O ; \mathbf{Q u}, \mathbf{Q v}, \mathbf{Q w})=\mathbf{Q} R$, the components of $\mathbf{T}$ are mapped into

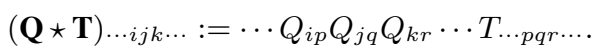

Let us now consider the alternating tensor $\epsilon_{i j k}$. It is easy to see that for $\mathbf{Q} \in \mathbf{O}(\mathbf{3})$ we have

$$
(\mathbf{Q} \star \epsilon)_{i j k}=Q_{i p} Q_{j q} Q_{k r} \epsilon_{p q r}=\operatorname{det}(\mathbf{Q}) \epsilon_{i j k} .
$$

The fact that the determinant of the orthogonal transformation $\mathbf{Q}$ appears in this relation means that the alternating tensor components are sensitive to space orientation. We shall say that they are pseudo-scalars or axial scalars and note them ${ }^{\epsilon}$ scalars. More precisely: as these scalars represent the alternating tensor, they are ${ }^{\epsilon}$ scalars. On the other hand, variables which are not sensitive to space orientation, in the sense that they transform as $\mathbf{T}$ in (25), are called polar variables.

Let us take another example: let $\mathbf{v} \in \mathbb{R}^{3}$ be a vector and let $\mathbf{V}$ be the antisymmetric tensor defined by $V_{i j}=\epsilon_{i j k} v_{k}$. Then $v_{k}=\frac{1}{2} \epsilon_{i j k} V_{i j}$. We write $\mathbf{v}=f(\mathbf{V})$. Consequently:

$$
f(\mathbf{Q} \star \mathbf{V})=\operatorname{det}(\mathbf{Q}) f(\mathbf{V}) .
$$

This relation means that $f$, which maps $\mathbf{V}$ into $\mathbf{v}$, is axial. It is therefore because the vector $\mathbf{v}$ represents a second-order antisymmetric tensor that we call it ${ }^{\epsilon}$ vector. We in fact say that $\mathbf{v}$ itself is axial, and note it ${ }^{\epsilon} \mathbf{v}$. By extension, we denote ${ }^{\epsilon} \mathbf{T}$ the tensors which do not transform as $\mathbf{T}$ in (25) but as the $\epsilon$ components in (26), translating that they are pseudo-tensors, or axial tensors.

Finally, for two tensors $\mathbf{T}$ (polar) and ${ }^{\epsilon} \mathbf{T}$ (axial) of same order and with the same components, we shall write

$$
\mathbf{T}={ }^{\epsilon} \operatorname{Id}\left({ }^{\epsilon} \mathbf{T}\right) .
$$

Such tensors are called twin tensors in the following.

\subsection{The piezomagnetic tensors symmetries}

$>$ From now on $\mathbb{R}^{3}$ is associated to a fixed orthonormal basis denoted $(\mathbf{i}, \mathbf{j}, \mathbf{k})$.

Let ${ }^{\epsilon}$ Magn be the space of third-order pseudo-tensors which are symmetric according to their first two indexes and odd according to time-reversal:

$$
{ }^{\epsilon} \mathbf{M a g n}:=\left\{{ }^{\epsilon} \mathbf{M}:{ }^{\epsilon} M_{i j k}={ }^{\epsilon} M_{j i k}, \mathfrak{R}{ }^{\epsilon} \mathbf{M}=-{ }^{\epsilon} \mathbf{M}\right\} .
$$

We also consider the set denoted Piez of all third-order polar tensors, symmetric according to their first two indexes:

$$
\text { Piez }:=\left\{\mathbf{P}: P_{i j k}=P_{j i k}\right\} \text {. }
$$

A tensor $\mathbf{P} \in \mathbf{P i e z}$ is called piezoelectric tensor.

In a piezomagnetic material, the magnetic polarization pseudo-vector ${ }^{\epsilon} b$ is connected to the stress tensor $\sigma$ via the relation

$$
{ }^{\epsilon} b_{k}={ }^{\epsilon} M_{i j k} \sigma_{i j},
$$

where ${ }^{\epsilon} \mathbf{M} \in{ }^{\epsilon} \mathbf{M a g n}$. When an (anti-)orthogonal transformation operates on a piezomagnetic tensor ${ }^{\epsilon} \mathbf{M}$ we thus have

$$
(\mathbf{Q}, \delta) \circledast{ }^{\epsilon} \mathbf{M}:=\delta \operatorname{det}(\mathbf{Q}) \mathbf{Q} \star{ }^{\epsilon} \mathbf{M}
$$

$$
\text { for }(\mathbf{Q}, \delta) \in \mathbf{O}^{\mu}(\mathbf{3}) \text {, }
$$


where $\star$ is defined in $(25)$.

We now consider the function which maps each element $(\mathbf{Q}, \delta)$ of $\mathbf{O}^{\mu}(\mathbf{3})$ into the automorphism of ${ }^{\epsilon} \mathbf{M a g n}$ defined by ${ }^{\epsilon} \mathbf{M} \mapsto(\mathbf{Q}, \delta) \circledast$ ${ }^{\epsilon} \mathbf{M}$. It is an action of $\mathbf{O}^{\mu}(\mathbf{3})$ on ${ }^{\epsilon} \mathbf{M a g n}$. The symmetry group $\mathcal{G}\left({ }^{\epsilon} \mathbf{M}\right)$ of ${ }^{\epsilon} \mathbf{M} \in{ }^{\epsilon} \mathbf{M a g n}$ thus takes the following form:

$$
\mathcal{G}\left({ }^{\epsilon} \mathbf{M}\right):=\left\{(\mathbf{Q}, \delta) \in \mathbf{O}^{\mu}(\mathbf{3}):(\mathbf{Q}, \delta) \circledast{ }^{\epsilon} \mathbf{M}={ }^{\epsilon} \mathbf{M}\right\}
$$

i.e. $\mathcal{G}\left({ }^{\epsilon} \mathbf{M}\right)$ collects all the orthogonal and anti-orthogonal operations that leave ${ }^{\epsilon} \mathbf{M}$ unchanged. For all ${ }^{\epsilon} \mathbf{M} \in{ }^{\epsilon} \mathbf{M a g n}$ we observe that the symmetry group $\mathcal{G}\left({ }^{\epsilon} \mathbf{M}\right)$ contains the element $(-\mathbf{I},+1)$ of $\mathbf{O}^{\mu}(\mathbf{3})$. Therefore, recalling (cf. $\S 2.5$.) that ${ }^{\mu} \mathbb{I d}$ is the subgroup $\{(\mathbf{I},+1),(-\mathbf{I},+1)\}$ and that $\oplus$ is the direct sum of subgroups in $\mathbf{O}^{\mu}(\mathbf{3})$, we necessarily have

$$
\mathcal{G}\left({ }^{\epsilon} \mathbf{M}\right)=\widetilde{g}\left({ }^{\epsilon} \mathbf{M}\right) \oplus{ }^{\mu} \mathbb{I} \mathrm{d},
$$

where $\widetilde{g}\left({ }^{\epsilon} \mathbf{M}\right)$ is defined by:

$$
\widetilde{g}\left({ }^{\epsilon} \mathbf{M}\right):=\left\{(\mathbf{Q}, \delta) \in \mathbf{S O}^{\mu}(\mathbf{3}):\right.
$$

$$
\left.(\mathbf{Q}, \delta) \circledast{ }^{\epsilon} \mathbf{M}=\delta \mathbf{Q} \star{ }^{\epsilon} \mathbf{M}={ }^{\epsilon} \mathbf{M}\right\},
$$

and where $\mathbf{S O}^{\mu}(3):=\mathbf{S O}(3) \times\{ \pm 1\}$

When now define the symmetry group $g(\mathbf{M})$ of an element $\mathbf{M} \in \mathbf{P i e z}$ by:

$$
g(\mathbf{M}):=\{\mathbf{Q} \in \mathbf{O}(\mathbf{3}): \mathbf{Q} \star \mathbf{M}=\mathbf{M}\} .
$$

We are going to show that the symmetry group $\mathcal{G}\left({ }^{\epsilon} \mathbf{M}\right)$ of the (axial) piezomagnetic tensor ${ }^{\epsilon} \mathbf{M}$ is intimately related to the symmetry group $g(\mathbf{M})=g\left({ }^{\epsilon} \operatorname{Id}\left({ }^{\epsilon} \mathbf{M}\right)\right)$ of its polar twin piezoelectric tensor. To this end, we introduce the isomorphism Iso defined by:

$$
\mathbf{S O}^{\mu}(\mathbf{3}) \ni(\mathbf{Q}, \delta) \stackrel{\text { Iso }}{\longmapsto} \quad \delta \mathbf{Q} \in \mathbf{O}(\mathbf{3}),
$$

and:

$$
\mathbf{O}(\mathbf{3}) \ni \mathbf{Q} \stackrel{\mathrm{Iso}^{-1}}{\longmapsto}(\operatorname{det}(\mathbf{Q}) \mathbf{Q}, \operatorname{det}(\mathbf{Q})) \in \mathbf{S O}^{\mu}(\mathbf{3}) .
$$

We then have:

$$
\begin{aligned}
\text { Iso }\left(\widetilde{g}\left({ }^{\epsilon} \mathbf{M}\right)\right) & =\left\{\mathbf{Q} \in \mathbf{O}(\mathbf{3}): \mathbf{Q} \star{ }^{\epsilon} \operatorname{Id}\left({ }^{\epsilon} \mathbf{M}\right)={ }^{\epsilon} \operatorname{Id}\left({ }^{\epsilon} \mathbf{M}\right)\right\} \\
& =\{\mathbf{Q} \in \mathbf{O}(\mathbf{3}): \mathbf{Q} \star \mathbf{M}=\mathbf{M}\} \\
& =g(\mathbf{M}) .
\end{aligned}
$$

Thanks to (34), the symmetry group $\mathcal{G}\left({ }^{\epsilon} \mathbf{M}\right) \subset \mathbf{O}^{\mu}(\mathbf{3})$ of the piezomagnetic pseudo-tensor ${ }^{\epsilon} \mathbf{M}$ is thus mapped into the symmetry group $g(\mathbf{M}) \subset \mathbf{O}(\mathbf{3})$ of its twin tensor, and vice versa. In other words, due to (37) and (38) the characterization of piezomagnetic tensors symmetries is strictly equivalent to the piezoelectric tensors symmetries one.

In this direction, we first of all observe that, by continuity, the groups $g(\mathbf{M})$ defined in (39) are closed subgroups of $\mathbf{O ( 3 )}$. To classify them in an understandable way, we build an equivalence relation as in $\S 2.2$. First of all, we note that $g(\mathbf{M})$ is conjugate through $\mathbf{Q} \in \mathbf{O}(\mathbf{3})$ to $g(\mathbf{Q} \star \mathbf{M})$ :

$$
g(\mathbf{Q} \star \mathbf{M})=\mathbf{Q} g(\mathbf{M}) \mathbf{Q}^{t}, \forall \mathbf{Q} \in \mathbf{O}(\mathbf{3}) .
$$

The idea is as follows: we will say that two tensors $\mathbf{M}_{1}$ and $\mathbf{M}_{2}$ in Piez are equivalent if there is an element $\mathbf{Q}$ of $\mathbf{O}(\mathbf{3})$ such that $g\left(\mathbf{M}_{1}\right)=$ $g\left(\mathbf{Q} \star \mathbf{M}_{2}\right)$. In other words, $\mathbf{M}_{1}$ and $\mathbf{M}_{2}$ are equivalent when their symmetry groups are conjugate:

$$
\mathbf{M}_{1} \stackrel{\star}{\sim} \mathbf{M}_{2} \Longleftrightarrow\{\exists \mathbf{Q} \in \mathbf{O}(\mathbf{3}):
$$

$$
\left.g\left(\mathbf{M}_{1}\right)=\mathbf{Q} g\left(\mathbf{M}_{2}\right) \mathbf{Q}^{t}\right\}
$$

Since $g(\mathbf{M})=g(-\mathbf{M})$, it is easily checked that : 


$$
\mathbf{M}_{1} \stackrel{\star}{\sim} \mathbf{M}_{2} \Longleftrightarrow\{\exists \mathbf{Q} \in \mathbf{S O}(3):
$$

$$
\left.g\left(\mathbf{M}_{1}\right)=\mathbf{Q} g\left(\mathbf{M}_{2}\right) \mathbf{Q}^{t}\right\}
$$

This relation translates the physical intuition that whenever two material bodies can be rigidly rotated so that their symmetry groups become identical, they share the same "symmetry".

Therefore, the equivalence classes of Piez which result from $\stackrel{\star}{\sim}$ are called symmetry classes and we note $\{\mathbf{M}\}$ the symmetry class of $\mathbf{M} \in$ Piez. In the same direction, for $\mathbb{G}$ a subgroup of $\mathbf{O ( 3 )}$, we note $\{\mathbb{G}\}$ the collection of all its conjugate among the subgroups of $\mathbf{O}(\mathbf{3})$. The question we ask now is: how many symmetry classes are there in Piez and which are there?

In order to give an accurate answer, we first of all point out some traditional results concerning the closed subgroups of $\mathbf{O}(\mathbf{3})$. It is well known (see for instance Forte \& Vianello, 1996, 1997; Golubitsky et al., 1988; Sivardière, 1995) that they fall in three categories (cf. Appendix A):

1. The closed subgroups of $\mathbf{S O}(3)$ or class $I$ closed subgroups: $\mathbb{I} d, \mathbb{Z}_{m}(m \geq 2), \mathbb{D}_{m}(m \geq 2), \mathcal{I}, \mathcal{O}, \mathcal{T}, \mathbf{S O}(\mathbf{2}), \mathbf{O ( 2 )}$ and $\mathbf{S O}(3)$.

2. The class II closed subgroups: $\mathbb{G} \oplus\{ \pm \mathbf{I}\}$, where $\mathbb{G}$ is a class I closed subgroup.

3. The class $I I I$ closed subgroups. They are not contained in $\mathbf{S O}(\mathbf{3})$ and do not contain $-\mathbf{I}: \mathbb{Z}_{2 m}^{-}(m \geq 1), \mathbb{D}_{2 m}^{d}(m \geq 2), \mathbb{D}_{m}^{z}(m \geq 2)$, $\mathcal{O}^{-}$and $\mathbf{O}(\mathbf{2})^{-}$.

Since the symmetry groups of the elements $\mathbf{M} \in \mathbf{P i e z}$ are closed subgroups of $\mathbf{O ( 3 )}$, each $\mathbf{M} \in \mathbf{P i e z}$ is therefore associated to a unique subgroup $\mathbb{G}$ defined above such that $\mathbb{G}$ is conjugate to $g(\mathbf{M})$. We write Piez $(\mathbb{G})$ for the collection of all tensors whose symmetry group is conjugate to $\mathbb{G}$ :

$$
\operatorname{Piez}(\mathbb{G}):=\{\mathbf{M} \in \mathbf{P i e z}: g(\mathbf{M}) \in\{\mathbb{G}\}\} .
$$

Obviously, we can apply the above results to tensors ${ }^{\epsilon} \mathbf{M} \in{ }^{\epsilon}$ Magn and to subgroups ${ }^{\mu} \mathbb{G} \subset \mathbf{O}^{\mu}(\mathbf{3})$. We then have:

$$
{ }^{\epsilon} \mathbf{M a g n}\left({ }^{\mu} \mathbb{G}\right):=\left\{{ }^{\epsilon} \mathbf{M} \in{ }^{\epsilon} \mathbf{M a g n}: \mathcal{G}\left({ }^{\epsilon} \mathbf{M}\right) \in\left\{{ }^{\mu} \mathbb{G}\right\}\right\} .
$$

In other words, the symmetry classes of Piez and ${ }^{\epsilon} \mathbf{M a g n}$ are related by the relations:

$$
\forall \mathbb{G} \subset \mathbf{O}(\mathbf{3})
$$

$$
\operatorname{Piez}(\mathbb{G})={ }^{\epsilon} \operatorname{Id}\left({ }^{\epsilon} \operatorname{Magn}\left(\operatorname{Iso}^{-1}(\mathbb{G}) \oplus^{\mu} \mathbb{I d}\right)\right),
$$

and:

$$
\forall^{\mu} \mathbb{G} \subset \mathbf{O}^{\mu}(\mathbf{3})
$$

$$
{ }^{\epsilon} \mathbf{M a g n}\left({ }^{\mu} \mathbb{G}\right)={ }^{\epsilon} \operatorname{Id}\left(\operatorname{Piez}\left(\operatorname{Iso}\left({ }^{\mu} \mathbb{G} \cap \mathbf{S O}^{\mu}(\mathbf{3})\right)\right)\right) .
$$

In the following, for any subgroup $\mathbb{G}$ of $\mathbf{O}(\mathbf{3})$, we will note ${ }^{\mu} \mathbb{G}$ the subgroup of $\mathbf{O}^{\mu}(\mathbf{3})$ defined by

$$
{ }^{\mu} \mathbb{G}:=\mathrm{Iso}^{-1}(\mathbb{G}) \oplus^{\mu} \mathbb{I d}
$$

Harmonic decomposition Any second-order tensor $\mathbf{E}$ can be seen as the sum of a symmetric tensor $\mathbf{S}=\frac{1}{2}\left(\mathbf{E}+\mathbf{E}^{t}\right)$ and of an antisymmetric tensor $\mathbf{W}=\frac{1}{2}\left(\mathbf{E}-\mathbf{E}^{t}\right)$ associated to an axial vector ${ }^{\epsilon} \mathbf{w}$ such that $W_{i j}=\epsilon_{i j k}{ }^{\epsilon} w_{k}$, where $\epsilon_{i j k}$ are the components of the alternating tensor. We note tr for the trace operator. The relation $\mathbf{S}=\mathbf{S}^{D}+\frac{1}{3}(\operatorname{tr} \mathbf{S}) \mathbf{I}=\mathbf{S}^{D}+\frac{1}{3}(\operatorname{tr} \mathbf{E}) \mathbf{I}$, where $\mathbf{S}^{D}$ is the deviatoric part of $\mathbf{S}$, points out an isomorphism $\phi$ between Lin and $\mathbf{D e v} \times \mathbb{R}^{3} \times \mathbb{R}$ where Dev is the space of second-order traceless and symmetric tensors. We therefore write:

$$
\text { Lin } \ni \mathbf{E} \approx\left(\mathbf{S}^{D},{ }^{\epsilon} \mathbf{w}, \frac{1}{3}(\operatorname{tr} \mathbf{E})\right) \in \operatorname{Dev} \times \mathbb{R}^{3} \times \mathbb{R}
$$

Moreover, according to (25) and (26) we have: 


$$
\mathbf{Q} \star \mathbf{E} \approx\left(\mathbf{Q} \star \mathbf{S}^{D}, \operatorname{det}(\mathbf{Q})\left(\mathbf{Q} \star{ }^{\epsilon} \mathbf{w}\right), \frac{1}{3}(\operatorname{tr} \mathbf{E})\right) .
$$

Thus the symmetry class of $\mathbf{E}$ verifies:

$$
g(\mathbf{E})=g\left(\mathbf{S}^{D}\right) \cap{ }^{\epsilon} g\left({ }^{\epsilon} \mathbf{w}\right),
$$

where $g(\mathbf{E})$ is defined as in (39) and where

$$
{ }^{\epsilon} g\left({ }^{\epsilon} \mathbf{w}\right)=\left\{\mathbf{Q} \in \mathbf{O}(\mathbf{3}): \mathbf{Q} \star{ }^{\epsilon} \mathbf{w}=\operatorname{det}(\mathbf{Q}){ }^{\epsilon} \mathbf{w}\right\} .
$$

The generalization of the correspondence $\phi$ as noted in (48) to tensors of any order (cf. Spencer (1970) and Appendix B) is called harmonic decomposition. A tensor is harmonic when it is totally symmetric (its components are unchanged under any permutations of indexes) and traceless (the trace with respect to any pair of indexes is null). Applied to Piez, this decomposition makes it possible to write:

$$
\text { Piez } \ni \mathbf{M} \approx\left(\mathbf{H},{ }^{\epsilon} \mathbf{C}, \nu, \mathbf{v}\right) \in \mathbf{H r m} \times{ }^{\epsilon} \operatorname{Dev} \times \mathbb{R}^{3} \times \mathbb{R}^{3}
$$

where Hrm is the space of third-order harmonic tensors and ${ }^{\epsilon}$ Dev the space of harmonic and axial second-order tensors. Some examples of this decomposition can be found in Forte \& Vianello (1997) and Schouten (1954). It is detailled in Appendix B. We then have:

$\forall \mathbf{Q} \in \mathbf{O}(\mathbf{3})$

$$
\mathbf{Q} \star \mathbf{M} \approx\left(\mathbf{Q} \star \mathbf{H}, \operatorname{det}(\mathbf{Q})\left(\mathbf{Q} \star{ }^{\epsilon} \mathbf{C}\right), \mathbf{Q} \star \nu, \mathbf{Q} \star \mathbf{v}\right),
$$

and thus:

$$
g(\mathbf{M})=g(\mathbf{H}) \cap{ }^{\epsilon} g\left({ }^{\epsilon} \mathbf{C}\right) \cap g(\nu) \cap g(\mathbf{v})
$$

where ${ }^{\epsilon} g\left({ }^{\epsilon} \mathbf{C}\right)$ follows from (51).

Cartan decomposition Let $\mathbf{r}=x \mathbf{i}+y \mathbf{j}+z \mathbf{k}$ be a vector of $\mathbb{R}^{3}$ and let $\mathcal{P}_{n}$ be the space of homogeneous polynomials of degree $n$ in the three variables $x, y$ and $z$. There is a classical isomorphism $\psi$ between $\mathcal{P}_{n}$ and $\mathbf{S y m}^{n}$, the space of totally symmetric tensors of order $n$ (cf. Forte \& Vianello, 1997):

$$
\mathbf{S y m}^{n} \ni \mathbf{T} \mapsto \psi(\mathbf{T}):=\mathbf{T}[\mathbf{r}, \mathbf{r}, \cdots, \mathbf{r}]
$$

$$
=T_{i_{1} i_{2} \cdots i_{n}} r_{i_{1}} r_{i_{2}} \cdots r_{i_{n}} \in \mathcal{P}_{n}
$$

where the convention of summation over repeated indexes is understood. Thus, the second-order symmetric tensor $\mathbf{S}=\left(S_{i j}\right)$ defined in the preceding paragraph is mapped into

$$
\psi(\mathbf{S})=S_{11} x^{2}+2 S_{12} x y+2 S_{13} x z
$$

$$
+S_{22} y^{2}+2 S_{23} y z+S_{33} z^{2}
$$

A polynomial $h \in \mathcal{P}_{n}$ is harmonic when $\Delta h=0$, where $\Delta$ is the Laplacian operator. We write $h \in \mathcal{H}_{n}$. It is easy to check that the space of harmonic tensors of order $n$ is isomorphic via $\psi$ to $\mathcal{H}_{n}$.

The isomorphism (55) enables us to extend the action $\star$ defined in (25) to $\mathcal{P}_{n}$ : for $p=\psi(\mathbf{T}) \in \mathcal{P}_{n}$ and $\mathbf{Q} \in \mathbf{O}(\mathbf{3})$, we define

$$
(\mathbf{Q} \star p)(x, y, z):=\mathbf{T}\left[\mathbf{Q}^{t} \mathbf{r}, \mathbf{Q}^{t} \mathbf{r}, \cdots, \mathbf{Q}^{t} \mathbf{r}\right]
$$

$$
=T_{i_{1} i_{2} \cdots i_{n}} r_{j_{1}} r_{j_{2}} \cdots r_{j_{n}} Q_{j_{1} i_{1}} Q_{j_{2} i_{2}} \cdots Q_{j_{n} i_{n}} .
$$


Moreover, the linear mapping $\psi$ is $\mathbf{O ( 3 )}$-invariant in the following sense

$\forall \mathbf{Q} \in \mathbf{O}(\mathbf{3}), \mathbf{Q} \star \psi(\mathbf{T})=\psi(\mathbf{Q} \star \mathbf{T})$

An essential property of $\mathcal{P}_{n}$ is that it is the direct sum of $\mathcal{H}_{n}$ and $\mathcal{Q}_{n}$, the space of polynomials which are multiples (by polynomials of $\mathcal{P}_{n-2}$ ) of $\rho:=x^{2}+y^{2}+z^{2}$ (it is a traditional result, cf. Golubitsky et al., 1988 p.109). Therefore, for each $p \in \mathcal{P}_{n}$, there exists a unique $q \in \mathcal{P}_{n-2}$ such that $h:=(p+\rho q) \in \mathcal{H}_{n}$. We call $h$ the harmonic part of $p$. The link with the harmonic decomposition is obvious: for $n=2$ and $p=\psi(\mathbf{S})$ we have $h=\psi\left(\mathbf{S}^{D}\right)$ and $q=\psi\left(\frac{1}{3} \operatorname{tr}(\mathbf{S})\right)$.

The goal is now to map harmonic polynomials (and consequently harmonic tensors) into spaces where the action $\star$ will become elementary. This purpose is achieved by the Cartan decomposition, which is an $\mathbf{S O}(2)$-invariant decomposition of $\mathcal{H}_{n}$. To give a preliminary idea of it, let us consider a second-order harmonic polynomial $f$ in the three variables $x, y$ and $z$. We then have $f \in \mathcal{H}_{2}$. It is clear that $f$ can be expressed on the following "basis":

$$
\begin{aligned}
u & :=z^{2}-\frac{1}{3} \rho \\
\left(s_{1}, t_{1}\right) & :=(x z, y z), \\
\left(s_{2}, t_{2}\right) & :=\left(x^{2}-y^{2}, 2 x y\right) .
\end{aligned}
$$

This decomposition can also be viewed as the decomposition of second-order deviatoric tensors on the following basis (up to constants):

$$
\begin{gathered}
\mathbf{U}=\left(\begin{array}{ccc}
1 & 0 & 0 \\
0 & 1 & 0 \\
0 & 0 & -2
\end{array}\right), \\
\mathbf{S}_{1}=\left(\begin{array}{lll}
0 & 0 & 1 \\
0 & 0 & 0 \\
1 & 0 & 0
\end{array}\right), \mathbf{T}_{1}=\left(\begin{array}{lll}
0 & 0 & 0 \\
0 & 0 & 1 \\
0 & 1 & 0
\end{array}\right), \\
\mathbf{S}_{2}=\left(\begin{array}{ccc}
1 & 0 & 0 \\
0 & -1 & 0 \\
0 & 0 & 0
\end{array}\right), \mathbf{T}_{2}=\left(\begin{array}{lll}
0 & 1 & 0 \\
1 & 0 & 0 \\
0 & 0 & 0
\end{array}\right),
\end{gathered}
$$

All these tensors are traceless, symmetric and translate very simply the action of orthogonal transformations which operate on them.

In the general case, let $w:=x+i y$ be a complex number. For $0 \leqslant l \leqslant n$ we note $s_{l}$ the harmonic part of $z^{n-l} \Re\left(w^{l}\right)$ and $t_{l}$ the harmonic part of $z^{n-l} \Im\left(w^{l}\right)$. In the following we write $u$ instead of $s_{0}$. We also notice that $t_{0}=0$. We finally define

$$
\begin{aligned}
K_{0} & :=\operatorname{vect}(u), \\
K_{l} & :=\operatorname{vect}\left(s_{l}, t_{l}\right), \quad 1 \leqslant l \leqslant n .
\end{aligned}
$$

The Cartan decomposition is stated as follows: the space $\mathcal{H}_{n}$ of harmonic polynomials of degree $n$ has an $\mathbf{S O}(2)$-invariant decomposition denoted

$$
K_{0} \oplus K_{1} \oplus \cdots \oplus K_{n}
$$

Moreover, each rotation $\mathbf{Q}(\mathbf{k}, \theta) \in \mathbf{S O}(2)$ about $\mathbf{k}$ of angle $\theta$ acts as the unit element on $K_{0}$ and like a rotation of angle $\theta$ on $K_{l}$ for $1 \leqslant l \leqslant n$ :

$$
\begin{aligned}
& \mathbf{Q}(\mathbf{k}, \theta) \star s_{l}=s_{l} \cos (l \theta)+t_{l} \sin (l \theta), \\
& \mathbf{Q}(\mathbf{k}, \theta) \star t_{l}=-s_{l} \sin (l \theta)+t_{l} \cos (l \theta) .
\end{aligned}
$$

Finally, $\mathbf{Q}(\mathbf{i}, \pi)$ acts as a reflection on $K_{l}(0 \leqslant l \leqslant n)$ : 


$$
\begin{aligned}
\mathbf{Q}(\mathbf{i}, \pi) \star u & =(-1)^{n} u, \\
\mathbf{Q}(\mathbf{i}, \pi) \star s_{l} & =(-1)^{n-l} s_{l}, \\
\mathbf{Q}(\mathbf{i}, \pi) \star t_{l} & =(-1)^{n-l+1} t_{l} .
\end{aligned}
$$

Arguments which amount to the above results are described in Golubitsky et al. (1988 p.109-111).

One of the immediate consequences of the Cartan decomposition is that to each $h \in \mathcal{H}_{n}$ correspond $2 n+1$ real numbers $\lambda, \alpha_{l}, \beta_{l}(1 \leqslant$ $l \leqslant n)$ such that

$$
h=\lambda u+\alpha_{l} s_{l}+\beta_{l} t_{l}, \quad 1 \leqslant l \leqslant n .
$$

The isomorphism $\psi$ defined in (55) enables us to transfer the Cartan decomposition from $\mathcal{H}_{n}$ into the space of harmonic tensors of degree $n$. The latter is then the direct sum of $n+1$ subspaces $\psi^{-1}\left(K_{l}\right)(0 \leqslant l \leqslant n)$ for which we use again the same symbol $K_{l}$ to simplify the notations.

If $n=3$, we consider an element $\mathbf{H} \in \mathbf{H r m}$ and write $\psi(\mathbf{H})=h=\lambda u+\alpha_{l} s_{l}+\beta_{l} t_{l}(1 \leqslant l \leqslant 3)$. Simple computations (see Forte \& Vianello, 1997; Golubitsky et al., 1988) show that a basis for $K_{l}(0 \leqslant l \leqslant 3)$ is given by:

$$
\begin{aligned}
u & =z^{3}-3 \rho z / 5, \\
\left(s_{1}, t_{1}\right) & =\left(z^{2} x-\rho x / 5, z^{2} y-\rho y / 5\right), \\
\left(s_{2}, t_{2}\right) & =\left(z\left(x^{2}-y^{2}\right), x y z\right) \\
\left(s_{3}, t_{3}\right) & =\left(x^{3}-3 x y^{2}, 3 x^{2} y-y^{3}\right) .
\end{aligned}
$$

Let $\mathbf{U}:=\psi^{-1}(u), \mathbf{S}_{l}:=\psi^{-1}\left(s_{l}\right)$ and $\mathbf{T}_{l}:=\psi^{-1}\left(t_{l}\right)$. For $\mathbf{H} \in \mathbf{H r m}$, we can write $\mathbf{H}=\mathbf{H}_{0}+\mathbf{H}_{1}+\mathbf{H}_{2}+\mathbf{H}_{3}$ where $\mathbf{H}_{0}:=\lambda \mathbf{U}$ and, for $1 \leqslant l \leqslant 3, \mathbf{H}_{l}:=\alpha_{l} \mathbf{S}_{l}+\beta_{l} \mathbf{T}_{l}$. The straightforward consequence of results (61)-(63) is the description and characterization of the symmetry groups of $\mathbf{H} \in \mathbf{H r m}$ through the properties of the "components" $\mathbf{H}_{l}$ (it will be said in the following that $\mathbf{H}_{l}$ is "horizontal" if it is a multiple of $\mathbf{S}_{l}$ and "vertical" if it is a multiple of $\mathbf{T}_{l}$ ):

Proposition 1. For a third-order harmonic tensor $\boldsymbol{H}=\boldsymbol{H}_{0}+\boldsymbol{H}_{1}+\boldsymbol{H}_{2}+\boldsymbol{H}_{3}$, we have:

(i) $g(\boldsymbol{H}) \supset \boldsymbol{S O}(2) \Longleftrightarrow \boldsymbol{H}=\boldsymbol{H}_{0} \Longleftrightarrow g(\boldsymbol{H}) \supset \boldsymbol{O}(\mathbf{2})^{-} \Longleftrightarrow g(\boldsymbol{H}) \supset \mathbb{D}_{k}^{z} \Longleftrightarrow g(\boldsymbol{H}) \supset \mathbb{Z}_{k}$, $(k \geq 4)$

(ii) $g(\boldsymbol{H}) \supset \boldsymbol{O}(2) \Longleftrightarrow \boldsymbol{H}=0 \Longleftrightarrow g(\boldsymbol{H}) \supset \mathbb{D}_{2 k}^{d}$

$\Longleftrightarrow g(\boldsymbol{H}) \supset \mathbb{Z}_{2 k}^{-} \Longleftrightarrow g(\boldsymbol{H}) \supset \mathbb{D}_{k},(k \geq 4) ;$

(iii) $g(\boldsymbol{H}) \supset \mathbb{Z}_{2} \Longleftrightarrow \boldsymbol{H}_{1}=\boldsymbol{H}_{3}=0$;

(iv) $g(\boldsymbol{H}) \supset \mathbb{D}_{2} \Longleftrightarrow \boldsymbol{H}$ is vertical in $K_{2}$

$\Longleftrightarrow g(\boldsymbol{H}) \supset \mathcal{T} \Longleftrightarrow g(\boldsymbol{H}) \supset \mathbb{D}_{4}^{d}$

$\Longleftrightarrow g(\boldsymbol{H}) \supset \mathcal{O}^{-}$

(v) $g(\boldsymbol{H}) \supset \mathbb{Z}_{3} \Longleftrightarrow \boldsymbol{H}_{1}=\boldsymbol{H}_{2}=0$;

(vi) $g(\boldsymbol{H}) \supset \mathbb{D}_{3} \Longleftrightarrow \boldsymbol{H}$ is horizontal in $K_{3}$;

(vii) $g(\boldsymbol{H}) \supset \mathbb{Z}_{2}^{-} \Longleftrightarrow \boldsymbol{H}_{0}=\boldsymbol{H}_{2}=0$;

(viii) $g(\boldsymbol{H}) \supset \mathbb{D}_{2}^{z} \Longleftrightarrow \boldsymbol{H}=\boldsymbol{H}_{0}+\boldsymbol{H}_{2}$ with $\boldsymbol{H}_{2}$ horizontal in $K_{2}$;

(ix) $g(\boldsymbol{H}) \supset \mathbb{Z}_{4}^{-} \Longleftrightarrow \boldsymbol{H}=\boldsymbol{H}_{2}$;

(x) $g(\boldsymbol{H}) \supset \mathbb{D}_{6}^{d} \Longleftrightarrow \boldsymbol{H}=\boldsymbol{H}_{3}$ with $\boldsymbol{H}_{3}$ horizontal in $K_{3}$;

(xi) $g(\boldsymbol{H}) \supset \mathbb{D}_{3}^{z} \Longleftrightarrow \boldsymbol{H}=\boldsymbol{H}_{0}+\boldsymbol{H}_{3}$ with $\boldsymbol{H}_{3}$ vertical in $K_{3}$

(xii) $g(\boldsymbol{H}) \supset \mathbb{Z}_{6}^{-} \Longleftrightarrow \boldsymbol{H}=\boldsymbol{H}_{3}$; 
Remark 1. A third-order harmonic tensor $\mathbf{H}$ has 7 components (three couples $\left(\alpha_{l}, \beta_{l}\right)_{1 \leq l \leq 3}$ and $\lambda$ ). The Cartan decomposition is particularly adapted to the determination of the number of constants which rise from a given symmetry. To take an example, part (iii) above indicates that $\mathbb{Z}_{2}$ symmetry requires three constants (one for $\mathbf{H}_{0}$ and two for $\mathbf{H}_{2}$ ). By applying this method to the other elements of the harmonic decomposition, we obtain the minimum number of constants of a tensor $\mathbf{M} \in \mathbf{P i e z}$ having a given symmetry. The determination of the optimal basis in which a given tensor is indeed written with this minimal number of constants requires a specific study however (cf. Berthaud, François \& Geymonat (1998) in the elasticity case).

Proof. - Relations concerning closed subgroups of SO(3) have been shown in Forte \& Vianello (1997). They are an immediate consequence of the Cartan decomposition. The other results easily come from Golubitsky et al. (1988 pp. 122 and 123)

\section{Symmetry classes of Piez We first show the}

Lemma 1. Let ${ }^{\epsilon} \boldsymbol{C} \in{ }^{\epsilon}$ Dev and $\boldsymbol{v} \in \mathbb{R}^{3}$. Then:

(a) ${ }^{\epsilon} g\left({ }^{\epsilon} \boldsymbol{C}\right)$ is conjugate to $\mathbb{D}_{2}, \mathbb{D}_{4}^{d}, \boldsymbol{O}(2)$ or $\boldsymbol{O}(3)$,

(b) $g(v)$ is conjugate to $\boldsymbol{O}(2)^{-}$or $\boldsymbol{O}(3)$.

Proof. - (a) It is an extension of Forte \& Vianello (1996, Theorem 3): if the three eigenvalues of ${ }^{\epsilon} \mathbf{C}$ are distinct and if none of them is zero then it does not exist an orthogonal transformation which maps ${ }^{\epsilon} \mathbf{C}$ into $-{ }^{\epsilon} \mathbf{C}$. We then are in the case of Forte \& Vianello (1996, Theorem 3). If one only of the eigenvalues is zero, then the two others are opposite and different from zero. The rotations of angle $\pi$ about the characteristic axes leave ${ }^{\epsilon} \mathbf{C}$ unchanged while an inversion of the characteristic axes associated to non zero eigenvalues maps ${ }^{\epsilon} \mathbf{C}$ into $-{ }^{\epsilon} \mathbf{C}$. This subgroup is conjugate to $\mathbb{D}_{4}^{d}$.

(b) If $\mathbf{v}$ is different from zero then there is a rotation $\mathbf{Q}$ such that $\mathbf{Q} \star \mathbf{v}$ is parallel to $\mathbf{k}$. Thus $g(\mathbf{v})=\mathbf{O ( 2 )}$

$>$ From proposition 1 and from Lemma 1 rises the

Corollary 1. Let $\boldsymbol{M} \in \boldsymbol{P i e z}$, we have:

(a) $g(\boldsymbol{M}) \supset \mathbb{Z}_{k} \Longleftrightarrow g(\boldsymbol{M}) \supset \boldsymbol{S O}(2),(k \geq 4)$,

(b) $g(\boldsymbol{M}) \supset \mathbb{D}_{k} \Longleftrightarrow \boldsymbol{M}=0 \Longleftrightarrow g(\boldsymbol{M}) \supset \mathbb{Z}_{2 k}^{-}$

$\Longleftrightarrow g(\boldsymbol{M}) \supset \mathbb{D}_{2 k}^{d},(k \geq 4)$,

(c) $g(\boldsymbol{M}) \supset \mathbb{Z}_{6}^{-} \Rightarrow\left\{\exists \boldsymbol{Q} \in \boldsymbol{S} \boldsymbol{O}(3): g(\boldsymbol{Q} \star \boldsymbol{M}) \supset \mathbb{D}_{6}^{d}\right\}$,

(d) $g(\boldsymbol{M}) \supset \mathbb{Z}_{2 k}^{-} \Longleftrightarrow g(\boldsymbol{M}) \supset \boldsymbol{O}(2),(k \geq 4)$,

(e) $g(\boldsymbol{M}) \supset \mathbb{D}_{k}^{z} \Longleftrightarrow g(\boldsymbol{M}) \supset \boldsymbol{O}(2)^{-},(k \geq 4)$,

(f) $g(\boldsymbol{M}) \supset \mathcal{T} \Longleftrightarrow g(\boldsymbol{M}) \supset \mathcal{O}^{-}$

Proof. - We just show part (c) with $\mathbf{M}=\left(\mathbf{H},{ }^{\epsilon} \mathbf{C}, \nu, \mathbf{v}\right)$ : if $g(\mathbf{M}) \supset \mathbb{Z}_{4}^{-}$then, according to part (a) of Lemma 1, we have ${ }^{\epsilon} g\left({ }^{\epsilon} \mathbf{C}\right) \supset \mathbf{S O}(\mathbf{2})$ ; moreover, $\nu=\mathbf{v}=\mathbf{0}$ and $\mathbf{H}=\mathbf{H}_{3}$ according to part (xii) of Proposition 1. But since there is a rotation $\mathbf{Q} \in \mathbf{S O}(\mathbf{2})$ which makes $\mathbf{H}_{3}$ vertical in $K_{3}$, we conclude by part (x) of Proposition 1 above and with Forte \& Vianello (1996, Lemma 2)

We consequently obtain trivially the

Corollary 2. Let $k \geq 4$ and $m \geq 3$, then Piez $\left(\mathbb{Z}_{k}\right)$,Piez $\left(\mathbb{D}_{k}\right)$, Piez $\left(\mathbb{D}_{2 k}^{d}\right)$, Piez $\left(\mathbb{D}_{k}^{z}\right)$,Piez $\left(\mathbb{Z}_{2 m}^{-}\right)$,Piez $(\mathcal{T})$, Piez $(\mathcal{O})$, Piez $(\mathcal{I})$ and Piez $(\operatorname{SO}(3))$ are empty. Moreover, $\boldsymbol{P i e z}(\boldsymbol{O}(\mathbf{3}))=\{\boldsymbol{0}\}$.

These results do not mean that there are no tensors in Piez that have, for example, a $\mathbb{Z}_{k}$ symmetry when $k \geq 4$, but such tensors then have necessarily a "higher" symmetry.

We finally have

Theorem 2. The following sets are not empty: Piez $(\mathbb{I} d)$, Piez $\left(\mathbb{Z}_{2}\right)$, Piez $\left(\mathbb{Z}_{3}\right)$, Magn $\left(\mathbb{Z}_{2}^{-}\right)$, Magn $\left(\mathbb{Z}_{4}^{-}\right)$, Piez $\left(\mathbb{D}_{2}^{z}\right), \operatorname{Piez}\left(\mathbb{D}_{3}^{z}\right)$, Piez $\left(\mathbb{D}_{2}\right)$, Piez $\left(\mathbb{D}_{3}\right)$, Piez $\left(\mathbb{D}_{4}^{d}\right)$, Piez $\left(\mathbb{D}_{6}^{d}\right)$, Piez $\left(\mathcal{O}^{-}\right)$, Piez $(\boldsymbol{S O}(2))$, Piez $(\boldsymbol{O}(2))$ and Piez $\left(\boldsymbol{O}(2)^{-}\right)$.

Proof. - The method consists in suitably choosing each element of the harmonic decomposition of $\mathbf{M}=\left(\mathbf{H},{ }^{\epsilon} \mathbf{C}, \nu, \mathbf{v}\right)$ in a similar way to that used in Forte \& Vianello $(1996,1997)$. Thus, by for example choosing a vertical tensor $\mathbf{H}$ in $K_{2}$ as the only non zero element in the harmonic decomposition of $\mathbf{M} \in \mathbf{P i e z}$, we have $g(\mathbf{M})=\mathcal{O}^{-}$ 


\section{Conclusions}

By considering the relations (45)-(47), the results obtained above are extended to ${ }^{\epsilon} \mathbf{M a g n}$. The containment relations between symmetry classes of ${ }^{\epsilon}$ Magn are presented on Fig. 1. This caracteristic tree shows the natural diagram of evolution of constitutive symmetries (relating to the behavior, i.e. to tensors (cf. Zheng \& Boelher, 1994)).

We recapitulate in Tab. 1 the bond between constitutive symmetries and microstructure symmetries (concerning notations cf. Bhagavantam (1996) and Birss (1996) for example). Each of the magnetic point groups $\underline{6}, \underline{\overline{6}}$ and $\underline{6} / \underline{m}$ are subgroups of ${ }^{\mu} \mathbb{Z}_{6}^{-}$. However, ${ }^{\mu} \mathbb{Z}_{6}^{-}$is not a constitutive symmetry group of the piezomagnetic phenomenon. This is a consequence of parts (c) of corollary 1 which states that piezomagnetic tensors cannot have ${ }^{\mu} \mathbb{Z}_{6}^{-}$symmetry without having a larger one. Therefore, with an appropriate rotation, any piezomagnetic tensor ${ }^{\epsilon} \mathbf{M}$ with symmetry group containing ${ }^{\mu} \mathbb{Z}_{6}^{-}$can be transformed into a piezomagnetic tensor with symmetry group containing ${ }^{\mu} \mathbb{D}_{6}^{d}$. This is why ${ }^{\mu} \mathbb{Z}_{6}^{-}$do not appear as piezomagnetic constitutive symmetry.

We then reduce to 15 the number of equivalence classes of piezomagnetic tensors, up to now considered to be 16.

\section{APPENDIXES}

\section{A - Closed subgroups of $\boldsymbol{O}(3)$}

As it was announced in $\S 3.4$, the closed subgroups of $\mathbf{O}(3)$ fall in three categories.

The class I subgroups are closed subgroups of $\mathbf{S O}(3)$ and are conjugate to one of the following groups:

- $\mathbb{I} d$, the trivial subgroup, formed by the unit element $\mathbf{I}$.

- $\mathbb{Z}_{m}$ (for $m \geq 2$ ), the set of rotations about $\mathbf{k}$ of angle multiple of $2 \pi / m$. It is a cyclic group with $m$ elements generated by $\mathbf{Q}(\mathbf{k}, 2 \pi / m)$.

- $\mathbb{D}_{m}$ (for $m \geq 2$ ), the dihedral group, generated by $\mathbb{Z}_{m}$ and $\mathbf{Q}(\mathbf{i}, \pi)$. It has $2 m$ elements.

- $\mathcal{T}$, the tetrahedral group containing the 12 symmetry elements of the tetrahedron.

- $\mathcal{O}$, the octahedral group containing the 24 symmetry elements of the octahedron or of the cube.

- $\mathcal{I}$, the symmetry group of icosahedron or dodecahedron. It has 60 elements.

- $\mathbf{S O}(2)$, the subgroup of rotations that leave $\mathbf{k}$ unchanged.

- $\mathbf{O ( 2 )}$, the subgroup of rotations which map $\mathbf{k}$ into $\pm \mathbf{k}$.

- $\operatorname{SO}(3)$.

The class II subgroups simply derive from class I subgroups (cf. $\S 3.3$ ).

As to class III subgroups, they have the characteristic not to be included in $\mathbf{S O}(3)$ and not to contain -I. They are defined in Golubitsky et al. (1988) for example. We detail here the structure of the $\mathbb{Z}_{2 m}^{-}(m \geq 1), \mathbb{D}_{2 m}^{d}(m \geq 2)$ and $\mathbb{D}_{m}^{z}(m \geq 2)$ subgroups.

The $\mathbb{Z}_{2}^{-}$subgroup is generated by

$$
\left(\begin{array}{ccc}
1 & 0 & 0 \\
0 & 1 & 0 \\
0 & 0 & -1
\end{array}\right) .
$$

Moreover, there are three nonconjugate subgroups of $\mathbf{O ( 3 )}$ isomorphic to $\mathbb{D}_{2 m}$, namely: $\mathbb{D}_{2 m}, \mathbb{D}_{2 m}^{z}$ and $\mathbb{D}_{2 m}^{d}$. The cyclic subgroup $\mathbb{Z}_{2 m}$ is present inside both $\mathbb{D}_{2 m}$ and $\mathbb{D}_{2 m}^{z}$. The $\mathbb{D}_{2 m}^{z}$ group is generated by $\mathbb{Z}_{2 m}$ and the reflection across the $x$-axis in the $(x, y)$-plane affected by

$$
\left(\begin{array}{ccc}
1 & 0 & 0 \\
0 & -1 & 0 \\
0 & 0 & 1
\end{array}\right)
$$

The cyclic subgroup of order $2 m$ in $\mathbb{D}_{2 m}^{d}$ is not the standard cyclic subgroup $\mathbb{Z}_{2 m} \subset \mathbf{S O}(3)$ but the class III subgroup $\mathbb{Z}_{2 m}^{-}$generated by the rotation

$$
\left(\begin{array}{ccc}
\cos \frac{\pi}{m} & \sin \frac{\pi}{m} & 0 \\
-\sin \frac{\pi}{m} & \cos \frac{\pi}{m} & 0 \\
0 & 0 & 1
\end{array}\right) .
$$

The reflection across the $x$-axis in $\mathbb{D}_{2 m}^{d}$ is the same as in $\mathbb{D}_{2 m}$.

\section{B - Harmonic Decomposition}

As announced in $\S 3.4 .1$, the harmonic decomposition is based on a method introduced by Spencer (1970). We apply it to the third-order tensors which are symmetric according to their first two indexes (in this direction, see Forte \& Vianello, 1997). We first point out the general step. 
Let $A_{i_{1} i_{2} i_{3} \cdots i_{n}}$ be the components of a tensor $\mathbf{A}$ of order $n$. We note $A_{\left(i_{1} i_{2} \cdots i_{r}\right) i_{r+1} \cdots i_{n}}$ the tensor obtained by symmetrization of $\mathbf{A}$ according to its first $r$ indexes. It is in fact the sum (divided by $r$ !) of the $r$ ! components obtained from $A_{i_{1} i_{2} i_{3} \cdots i_{n}}$ by applying all the permutations to its first $r$ ! indexes. The fundamental relation due to Spencer (1970) is:

$$
\begin{aligned}
& r A_{\left(i_{1} i_{2} \cdots i_{r-1}\right) i_{r} i_{r+1} \cdots i_{n}}=r A_{\left(i_{1} i_{2} \cdots i_{r}\right) i_{r+1} \cdots i_{n}} \\
& +\epsilon_{i_{1} i_{r} i_{s}} \epsilon_{i_{p} i_{q} i_{s}} A_{\left(i_{p} i_{2} i_{3} \cdots i_{r-1}\right) i_{q} i_{r+1} \cdots i_{n}} \\
& +\epsilon_{i_{2} i_{r} i_{s}} \epsilon_{i_{p} i_{q} i_{s}} A_{\left(i_{p} i_{1} i_{3} \cdots i_{r-1}\right) i_{q} i_{r+1} \cdots i_{n}}
\end{aligned}
$$

$$
+\cdots+\epsilon_{i_{r-1} i_{r} i_{s}} \epsilon_{i_{p} i_{q} i_{s}} A_{\left(i_{p} i_{1} i_{3} \cdots i_{r-2}\right) i_{q} i_{r+1} \cdots i_{n}}
$$

where $\epsilon_{i_{1} i_{2} i_{3}}$ are the components of the alternating tensor.

We define

$$
B_{i_{1} i_{2} i_{3} \cdots i_{r-2} i_{r+1} \cdots i_{n} i_{s}}:=\epsilon_{i_{p} i_{q} i_{s}} A_{\left(i_{p} i_{1} i_{2} \cdots i_{r-2}\right) i_{q} i_{r+1} \cdots i_{n}} .
$$

Thus $A_{\left(i_{1} i_{2} \cdots i_{r-1}\right) i_{r} \cdots i_{n}}$ is the sum of the symmetrization of the tensor $\mathbf{A}=A_{i_{1} i_{2} i_{3} \cdots i_{n}}$ with respect to its first $r$ ! indexes and some terms obtained by contraction of $B_{i_{1} i_{2} i_{3} \cdots i_{r-2} i_{r+1} \cdots i_{n} i_{s}}$ with the alternating tensor. These terms are therefore pseudo-tensors. Applying by recurrence the equality above, we are finally able to express $A_{i_{1} i_{2} i_{3} \cdots i_{n}}$ as a sum of (pseudo-)tensors totally symmetric of order equal or less than $n$, suitably contracted with isotropic tensors.

Let $\mathbf{M} \in$ Piez. By definition, we have $\mathbf{M}=M_{(i j) k}$. We note it $M_{i j k}=M_{(i j) k}$. Applying the relation above we find:

$$
3 M_{(i j) k}=3 M_{(i j k)}+\epsilon_{i k p} \epsilon_{a b p} M_{a j b}+\epsilon_{j k p} \epsilon_{a b p} M_{a i b} .
$$

Thus $M_{(i j k)}$ is a third-order totally symmetric tensor. It again breaks up into a third-order harmonic tensor $\mathbf{H}$ and a vector $\nu \in \mathbb{R}^{3}$. In addition, by letting ${ }^{\epsilon} C_{j p}=\epsilon_{a b p} M_{a j b}$, we obtain:

$$
2{ }^{\epsilon} C_{i j}=2{ }^{\epsilon} C_{(i j)}+\epsilon_{i j p} v_{p},
$$

where $v_{p}$ are the components of a vector $\mathbf{v} \in \mathbb{R}^{3}$.

It is thus seen that ${ }^{\epsilon} C_{(i j)}$ is a second-order symmetric pseudo-tensor. Simple calculations show that it is traceless: ${ }^{\epsilon} \mathbf{C}$ is harmonic.

We then show that any tensor $\mathbf{M} \in \mathbf{P i e z}$ corresponds to a quadruplet including a third -order harmonic tensor $\mathbf{H}$ (with 7 components), a second-order harmonic pseudo-tensor ${ }^{\epsilon} \mathbf{C}$ (5 components) and two vectors $\mathbf{v}$ and $\nu$ (3 components each). We therefore verify that $\mathbf{M}=$ $M_{(i j) k} \in$ Piez has 18 components. This is the reason why such tensors are most of the time presented in the form of a $3 \times 6$ matrix. This correspondence is linear like every operations that intervene in the harmonic decomposition. Its kernel is $\{0\}$ : it is consequently an isomorphism. The property of $\mathbf{S O}(3)$-invariance according to the $\star$ action rises simply from the following equality, due to (25) and (26):

$\forall \mathbf{Q} \in \mathbf{S O}(\mathbf{3}), \mathbf{Q} \star \mathbf{M}$

$$
=\left(\mathbf{Q} \star \mathbf{H}, \operatorname{det}(\mathbf{Q}) \mathbf{Q} \star{ }^{\epsilon} \mathbf{C}, \mathbf{Q} \star \mathbf{v}, \mathbf{Q} \star \nu\right) .
$$


Table 1. Constitutive symmetries of the piezomagnetic phenomenon related to the magnetic point groups which give them birth

\begin{tabular}{|c|c|}
\hline $\begin{array}{l}\text { Constitutive } \\
\text { symmetries } \\
\left(G_{\text {property }}\right)\end{array}$ & Magnetic point groups $\left(G_{\text {point }}\right)$ \\
\hline${ }^{\mu} \mathrm{Id}$ & $1, \overline{1}$ \\
\hline${ }^{\mu} \mathbb{Z}_{2}^{-}$ & $\underline{\underline{m}}, \underline{2}, \underline{2} / \underline{m}$ \\
\hline${ }^{\mu} \mathbb{Z}_{4}^{-}$ & $\underline{4}, \underline{\overline{4}}, \underline{4} / m$ \\
\hline${ }^{\mu} \mathbb{Z}_{2}$ & $m, 2,2 / m$ \\
\hline${ }^{\mu} \mathbb{Z}_{3}$ & $3, \overline{3}$ \\
\hline${ }^{\mu} \mathbb{D}_{2}^{z}$ & $\underline{2} m \underline{m}, 2 \underline{m m}, \underline{222}, m \underline{m m}$ \\
\hline${ }^{\mu} \mathbb{D}_{2}$ & $2 \mathrm{~mm}, 222, \mathrm{mmm}$ \\
\hline${ }^{\mu} \mathbb{D}_{3}^{z}$ & $3 \underline{2}, 3 \underline{m}, \overline{3} \underline{m}$ \\
\hline${ }^{\mu} \mathbb{D}_{3}$ & $32, \overline{3 m}, \overline{3 m}$ \\
\hline${ }^{\mu} \mathbb{D}_{4}^{d}$ & $\underline{4} 2 \underline{2}, \underline{4} m \underline{m}, \underline{\overline{4}} 2 \underline{m}, \underline{\overline{4}} \underline{2} m, \underline{4} / m m \underline{m}$ \\
\hline${ }^{\mu} \mathbb{D}_{6}^{d}$ & $\begin{array}{l}\underline{6}, \underline{\overline{6}}, \underline{6} / \underline{m}, \underline{6} 2 \underline{\underline{m}} m \underline{m}, \underline{6 m} m, \underline{\overline{6} m} 2, \underline{\overline{6}} m \underline{2},\end{array}$ \\
\hline${ }^{\mu} \mathcal{O}^{-}$ & $23, m 3, \underline{4} 32, \underline{4} 3 \underline{m}, m 3 \underline{m}$ \\
\hline${ }^{\mu} \mathbf{S O}(2)$ & $4, \overline{4}, 4 / m, 6, \overline{6}, 6 / m$ \\
\hline${ }^{\mu} \mathbf{O}(\mathbf{2})$ & $\begin{array}{l}422,4 m m, \quad \overline{4} 2 m, \quad 4 / m m m, \quad 622, \\
6 m m, \overline{6} m 2,6 / m m m\end{array}$ \\
\hline${ }^{\mu} \mathbf{O}(2)^{-}$ & $\begin{array}{l}4 \underline{22}, 4 \underline{m m}, \underline{\overline{4} 2 m}, 4 / m \underline{m m}, 6 \underline{22}, \\
6 \underline{m m}, 6 \underline{m 2}, 6 / m \underline{m m}\end{array}$ \\
\hline
\end{tabular}

Fig. 1. Characteristic tree of ${ }^{\epsilon}$ Magn symmetry classes.

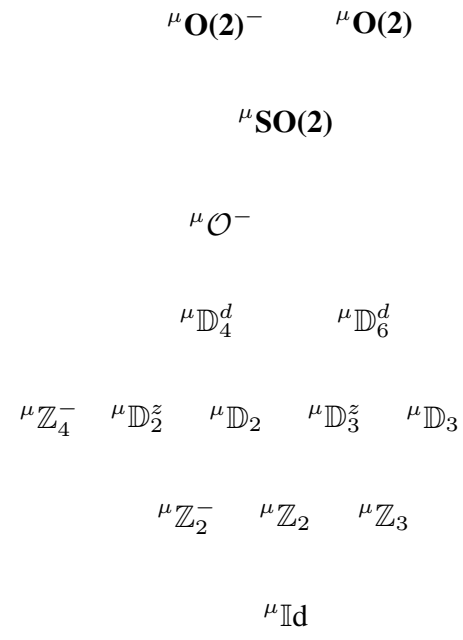

\section{References}

Berthaud Y. \& François M. \& Geymonat G. (1998). Int. J. Solids Structures 35, 4091-4106.

Bhagavantam S. (1966). Crystal Symmetry and Physical Properties, Academic Press.

Birss R. (1966). Symmetry and Magnetism, North Holland.

Coxeter H.S.M. (1989). Introduction to Geometry, John Wiley and Sons.

Forte S. \& Vianello M. (1996). J. Elasticity 43, 81-108.

Forte S. \& Vianello M. (1997). Int. J. Engng Sci. 35, 1317-1326.

Geymonat G. \& Weller T. (2002). C. R. Acad. Sci. Série I 335, 847-852.

Golubitsky M. \& Stewart I. \& Schaeffer D.G. (1988). Singularities and Groups in Bifurcation Theory, Vol. 2, Springer Verlag. Huo Y.-Z. \& Del Piero G. (1991). J. Elasticity 25, 203-246.

Landau L. \& Lifchitz E. (1969). Électrodynamique des Milieux Continus, Éditions Mir. 
Laughlin D.E. \& Willard M.A. \& McHenry M.E. (2000). The Mineral, Metal and Material Society, 121-137.

Nye J.F. (1987) Reprint. Physical Properties of Crystals: their representation by tensors and matrices, Clarendon Press.

Pitteri M. \& Zanzotto G. (1996). Acta Cryst. A52, 830-838.

Pitteri M. \& Zanzotto G. (1998). Acta Cryst. A54, 359-373.

Pitteri M. \& Zanzotto G. (2003). Continuum Models for Phase Transitions and Twinning in Crystals, Chapman \& Hall/CRC.

Schouten J.A. (1954). Tensor Analysis for Physicists, Clarendon Press.

Shubnikov A.V. (1964). Colored Symmetry, Pergamon Press.

Sirotine Y. \& Chaskolskaïa M. (1984). Fondements de la Physique des Cristaux, Éditions Mir.

Sivardière J. (1995). La Symétrie en Mathématiques, Physique et Chimie, Presses Universitaires de Grenoble.

Spencer A.J.M. (1970). Int. J. Engng Sci. 8, 475-481.

Weigel D. (1972). Cristallographie et Structure des Solides, Tome 1: algèbre et géométrie cristalline et moléculaire, Masson.

Weyl H. (1952). Symmetry, Princeton University Press.

Yale P.B. (1968). Geometry and Symmetry, Holden-Day.

Zheng Q.-S. \& Boelher J.P. (1994). Acta Mechanica 102, 73-89. 